\title{
Prospective surveillance of sympathetic ophthalmia in the UK and Republic of Ireland
}

\author{
Dara J Kilmartin, Andrew D Dick, John V Forrester
}

\begin{abstract}
Aims-To establish current epidemiological data, risks, and interventional outcomes of newly diagnosed sympathetic ophthalmia (SO).

Methods-Prospective surveillance took place of all permanently employed ophthalmologists in the UK and Republic of Ireland by a monthly reporting card through the British Ophthalmological Surveillance Unit. Case ascertainment was made of newly diagnosed SO from July 1997 and questionnaire data were returned at baseline, 6 months, and 1 year after diagnosis.

Results-23 patients with newly diagnosed SO were recruited over 15 months, corresponding to a minimum estimated incidence of 0.03/100 000. Baseline data were available on 18 patients, in whom SO occurred after surgery in 11 patients, after retinal surgery alone in six patients, and after accidental trauma in seven patients. 12 of the 16 patients with 1 year follow up had a visual acuity of $6 / 12$ or better. Good visual outcome was related to prompt and adequate systemic immunosuppressive therapy.

Conclusions-The incidence of sympathetic ophthalmia is very low. The main current risk is surgery, particularly retinal surgery, but visual prognosis is good if early diagnosis is made and rapid, adequate immunotherapy is commenced. (Br F Ophthalmol 2000;84:259-263)
\end{abstract}

Sympathetic ophthalmia (SO) is an uncommon bilateral granulomatous panuveitis following uveal trauma to one eye. Although SO does not have a large direct public health impact, with previous estimates of prevalence varying from $0.1 \%$ to $0.3 \%$ following accidental ocular trauma ${ }^{1-3}$ and $0.02 \%$ following ocular surgery, ${ }^{4-6}$ the possibly increasing causative role of ocular surgery in SO is an important epidemiological development. ${ }^{7}$ Sympathetic ophthalmia is also important because it acts as a classic model for autoimmune disease where posterior segment intraocular inflammation develops after sensitisation to previously sequestered ocular antigens. ${ }^{8-11}$ Previous studies of SO have been exclusively retrospective, with clinical data often more than 10 years old, and may not reflect the benefits of modern microsurgical techniques and immunosuppression. ${ }^{72-14}$ Retrospective case series have found that higher SO risks include accidental ocular trauma rather than ocular surgery, male sex, and biphasic peaks in children and the elderly because of the greater incidence of accidental trauma and ocular surgery respectively. ${ }^{12-14}$ However, a recent report found that SO incidence may be increasing after surgery, in female patients, and in elderly patients. ${ }^{7}$ The indications and timing of enucleation in the management of SO also remain controversial. The aim of this study was to prospectively recruit all newly diagnosed cases of SO in Britain and Ireland to establish current epidemiological data including incidence, current risks, treatment modalities, and outcomes.

\section{Materials and methods}

Through the British Ophthalmological Surveillance Unit (BOSU), all permanently employed ophthalmologists in the UK were sent a monthly reporting card requesting notification of a case of newly diagnosed SO from July 1997 to September 1998 inclusive. For the last 3 months of the study, ophthalmologists in the Republic of Ireland were included in the scheme. Ophthalmologists were identified from the databases of the Royal College of Ophthalmologists and Irish College of Ophthalmologists respectively. Sympathetic ophthalmia was included as one of four uncommon disease entities on the reporting card and ophthalmologists were also asked to indicate that there was no new case to report. Following positive notification to the BOSU, every reporting ophthalmologist was sent a detailed questionnaire by the study investigators at baseline, 6 months, and 1 year after diagnosis. Inclusion criteria included a definite history of ocular trauma or surgery and bilateral (or unilateral signs if enucleation had been performed) intermediate uveitis/posterior uveitis/ panuveitis consistent with SO. Histology was not required for diagnosis. All patients signed the informed consent form approved by the joint ethics committee of the University of Aberdeen and Grampian Health Board and the study adhered to the tenets of the Declaration of Helsinki. Data were returned after informed consent on age, sex, nature of trauma, visual acuity, ophthalmoscopy including anterior chamber and vitreal activity, enucleation, and immunosuppressive therapy. The results of histology (if enucleation performed) were also requested.

\section{Results}

From July 1997 to September 1998 inclusive, 55 reports of patients with newly diagnosed SO were made to the BOSU from 876 ophthalmologists in the UK and 85 ophthalmologists in the Republic of Ireland. Of these, 12 were 
duplicate reports, eight were pre-established one centre confirmed definite new cases (and were included for incidence estimation because of the expertise of the reporting ophthalmologist) but further information was not made available, five reports resulted in no reply, and two reports did not satisfy inclusion criteria for diagnosis. Twenty three valid cases were reported, of whom 16 had their diagnosis confirmed by a uveitis subspecialist and seven had been diagnosed by a medical retina subspecialist. Data were therefore available on 18 patients at baseline, 16 patients at 6 months and 1 year follow up after diagnosis. Two patients were lost to follow up, one each from Scotland and Northern Ireland, but were included in the estimation of incidence. There were no cases reported (average regional reporting card response rates given in parentheses) from the West Midlands (63\%), Wales $(58 \%)$, or the Channel Islands (52\%). For the first 3 months of patient recruitment, the average monthly response rate was $55 \%$ but rose to an average of $64 \%$ for the following 12 months of recruitment (Table 1).

INCIDENCE AND RISKS FOR SO

For the purposes of incidence estimation, cases only reported in the last consecutive 12 months of recruitment were included where the average response rate was consistently greater than or equal to $60 \%$. The response rate in the first 3 months was consistently less than this and therefore five cases reported in the first 3 months were excluded. The one case reported from the Republic of Ireland was also excluded from incidence estimation as recruitment only began there in the last 3 months of the study. This yielded 17 patients drawn from surveilminimum estimated incidence of $0.03 / 100000$ of the general UK population. More cases of SO were diagnosed in spring and autumn than summer or winter, with six $(33 \%)$ cases diagnosed in the combined months of September and October 1997 and seven (38\%) cases diagnosed from February to May 1998 inclucases, five were clerical errors, five reports from lance of 59.0 million in the UK, ${ }^{15}$ producing a

sive. No sex predominance was observed, with nine males and nine females diagnosed. The mean age at diagnosis of SO was 56.1 (SD 18.5) years (Table 1). New cases occurred more frequently in the elderly, with nine $(50 \%)$ cases in those aged 65 years or over at diagnosis compared with only one $(6 \%)$ child with newly diagnosed SO.

SURGERY IS THE COMMONEST CAUSE OF SO Ocular surgery, predominantly retinal surgery, was the commonest injury. Ocular surgery was the initiating injury in $11(61 \%)$ patients and the sole cause of injury in $10(56 \%)$ patients. Trauma, exclusively penetrating ocular injury, was the sole cause of injury in six (33\%) patients. Where ocular surgery was the sole cause of injury, all 10 patients had undergone at least one retinal surgical procedure, usually pars plana vitrectomy, with a median of two ocular surgical procedures and 1.5 (range 1-4) retinal surgical procedures performed in each patient (Table 1). Retinal surgery alone was the initiating injury in six (33\%) patients, with three of these patients having undergone just one pars plana vitrectomy. As most patients had more than one ocular injury event (accidental trauma or surgery), the timing from both initial and final injury to disease onset is described. The median time from initial injury to onset of signs or symptoms was 12 months (range 3 weeks to 43 years) and the median time from final injury to onset of signs or symptoms was 5 months (range 3 weeks to 9 years). The time from initial injury to disease onset was less than 1 year in eight (44\%) patients, and the time from final injury to disease onset was less than 1 year in $14(78 \%)$ patients.

The median time from onset of symptoms to diagnosis was 2 weeks (range 3 days to 8 months) and was less than 2 months in 13 $(72 \%)$ patients. Seven $(39 \%)$ patients had a diagnosis of sympathetic ophthalmia made within 1 week of onset of clinical symptoms. Most patients had moderate or severe panuveitis at presentation. Dalen-Fuchs nodules were present in eight $(44 \%)$ patients (Table 2).

Table 1 Patient demographics and nature of injury

\begin{tabular}{|c|c|c|c|c|}
\hline Patient No/sex & $\begin{array}{l}\text { Region/response } \\
\text { rate }(\%)^{*}\end{array}$ & $\begin{array}{l}\text { Age (at Dxt, initial } \\
\text { injury) (years) }\end{array}$ & Type of injury & Surgical proceduret \\
\hline $1 / \mathrm{F}$ & $\mathrm{A} \& \mathrm{O}(58)$ & 73,73 & Surgery, trauma & Trabeculectomy \\
\hline $2 / \mathrm{F}$ & S\&W (72) & 75,67 & Surgery & Ext RD, cataract \\
\hline $3 / \mathrm{F}$ & $N \& Y(61)$ & 66,65 & Surgery & PPV RD \\
\hline $4 / M$ & $\mathrm{~T}(57)$ & 71,69 & Surgery & Cataract + Ant Vity, Ext RD \\
\hline $5 / \mathrm{F}$ & NW (56) & 73,72 & Surgery & Ext beam DTX, PPV $\times 2$, Enucl \\
\hline $6 / M$ & NI (61) & 40,40 & Surgery & PPV \\
\hline $7 / \mathrm{M}$ & $\mathrm{S}(72)$ & 40,10 & Trauma & \\
\hline $8 / M$ & NT (57) & 40,40 & Trauma & \\
\hline $9 / M$ & $\mathrm{~S}$ & 44,43 & Trauma & \\
\hline $10 / \mathrm{M}$ & ST (64) & 66,66 & Surgery & PPV RD \\
\hline $11 / \mathrm{M}$ & S\&W & 68,66 & Surgery & PPV RD \\
\hline $12 / \mathrm{F}$ & NI & 14,14 & Trauma & \\
\hline $13 / \mathrm{M}$ & S\&W & 31,30 & Trauma & \\
\hline $14 / \mathrm{F}$ & NW & 71,69 & Surgery & Ext RD ×2 \\
\hline $15 / \mathrm{F}$ & ROI (31) & 62,60 & Surgery & $\mathrm{PPV} R \mathrm{RD} \times 3$ \\
\hline $16 / \mathrm{F}$ & ST & 74,70 & Surgery & Ext RD, PPV RD, Cyclodiodetx \\
\hline $17 / \mathrm{M}$ & NT & 47,4 & Trauma, surgery & PPV RD ×2 \\
\hline $18 / \mathrm{F}$ & $\mathrm{N} \& \mathrm{Y}$ & 52,52 & Trauma & \\
\hline
\end{tabular}

${ }^{\star} \mathrm{A} \& \mathrm{O}=$ Anglia and Oxford, $\mathrm{S} \& \mathrm{~W}=$ South and West, $\mathrm{N} \& \mathrm{Y}=$ Northern and Yorkshire, $\mathrm{T}=$ Trent, $\mathrm{NW}=$ North West, NI $=\mathrm{Northern}$ Ireland, $\mathrm{S}=$ Scotland, NT $=$ North Thames, $\mathrm{ST}=$ South Thames, ROI $=$ Republic of Ireland. $+\mathrm{Dx}=$ diagnosis, Ext $=$ external, $\mathrm{RD}=$ retinal detachment repair, Cataract $=$ cataract extraction, Ant Vity $=$ anterior vitrectomy, DTX $=$ radiotherapy, PPV $=$ pars plana vitrectomy, Enucl $=$ enucleation, Cyclodiodet $=$ cyclodiodetherapy. 
Table 2 Clinical features at presentation

\begin{tabular}{ll}
\hline & No (\%) of patients $(n=18)$ \\
\hline Moderate/severe panuveitis & $11(61)$ \\
Moderate/severe anterior chamber inflammation & $11(61)$ \\
No anterior chamber inflammation & $1(6)$ \\
Dalen-Fuchs nodules & $8(44)$ \\
Choroidal neovascularisation & $1(6)$ \\
Extraocular signs & $1(6)$
\end{tabular}

Choroidal neovascularisation, in one $(6 \%)$ patient (patient 5), and extraocular signs (sensorineural deafness), in one $(6 \%)$ patient (patient 1), were both observed. Patient 17 was initially misdiagnosed as multiple evanescent white dot syndrome.

TREATMENT AND OUTCOME

Systemic corticosteroids (CST) were instituted in $17(94 \%)$ of 18 patients diagnosed, and within 2 weeks of symptom onset in eight (44\%) patients. On follow up of 16 patients, additional systemic immunosuppression was started in $11(69 \%)$ patients, with cyclosporin A (CsA) in eight $(50 \%)$ patients, azathioprine (Aza) in three (19\%), and mycophenolate mofetil (MMF) in one (6\%) patient. Maintenance dosage was CST: 0.15 (SD 0.11) mg/kg/ day, CsA: 3.0 (1.0) mg/kg/day, Aza: 1.8 (0.6) $\mathrm{mg} / \mathrm{kg} / \mathrm{day}$, and MMF: $2 \mathrm{~g}$ daily. Most of the patients followed up received either CST monotherapy (six (38\%) patients), or dual therapy with CST and CsA (five (31\%) patients), with other patients treated with CST and Aza (three patients), CST, CsA, and Aza (one patient), CST, CsA, and MMF (one patient), and CsA monotherapy (one patient).

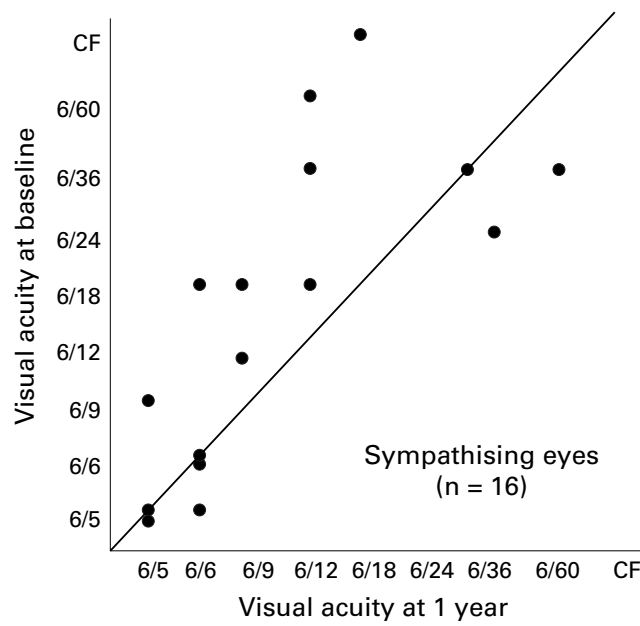

Figure 1 Visual outcome 1 year after diagnosis.

Table 3 Treatment and relation to visual outcome at 1 year follow up

\begin{tabular}{|c|c|c|c|}
\hline \multirow[b]{2}{*}{ Visual outcome } & \multicolumn{3}{|c|}{ No (\%) of patients } \\
\hline & $\begin{array}{l}V A \geqslant 6 / 12 \\
(n=12)\end{array}$ & $\begin{array}{l}V A \leqslant 6 / 60 \\
(n=1)\end{array}$ & $\begin{array}{l}\text { VA improvement } \\
\geqslant 2 \text { Snellen lines } \\
(n=7)\end{array}$ \\
\hline Adequate and rapid medication ${ }^{\star}$ & $10(83)$ & 0 & 6 \\
\hline Enucleation & $3(25)$ & 1 & 1 \\
\hline \multicolumn{4}{|c|}{ Time interval from symptoms to diagnosis } \\
\hline$<2$ months & $10(83)$ & 1 & 5 \\
\hline$\geqslant 2$ months & $2(17)$ & 0 & 2 \\
\hline
\end{tabular}

*Active disease treated with immunosuppression/ prednisolone $\geqslant 0.33 \mathrm{mg} / \mathrm{kg} /$ day less than 2 weeks after the onset of symptoms.
Most patients had either improved or maintained visual acuity at 1 year after diagnosis (Fig 1). Twelve (75\%) of 16 patients had a 1 year visual acuity of $0.5(6 / 12)$ or better, and visual acuity improved by at least 2 Snellen lines in seven $(44 \%)$ patients. Only one $(6 \%)$ patient (patient 5) had a visual acuity of 0.1 (6/60), from subfoveal choroidal neovascularisation, and no patient had worse visual acuity than this level 1 year after diagnosis. Good visual outcome was associated with adequate immunotherapy. Using criteria defined by Chan $e t a l^{11}$ for comparison, patients were considered to be inadequately treated if they had active disease that was treated with no immunosuppressive agent (including CST) or with systemic prednisolone at a dose lower than $0.33 \mathrm{mg} / \mathrm{kg} / \mathrm{day}$, or if treatment was started more than 2 weeks after the onset of symptoms. Good visual acuity (better than or equal to 6/12), and improvement from initial visual acuity by at least 2 Snellen lines, was related to adequate and rapid systemic therapy and a shorter time interval (less than 2 months) from symptom onset to diagnosis (Table 3).

ENUCLEATION IS NOT RELATED TO BETTER VISUAL OUTCOME

Enucleation was performed in five $(27 \%)$ patients, at a median of 4 weeks after diagnosis. Despite enucleation for recurrent choroidal melanoma in one patient (patient 5), SO developed 6 months later. Reasons for enucleation were varied and included intervention to reduce inflammation in the sympathising eye (two patients), to remove a blind painful eye (one patient), to confirm the diagnosis of SO (one patient), and recurrence of choroidal melanoma (one patient). Histology of the four exciting eyes available showed typical nonnecrotising granulomatous uveal inflammation in three eyes. There were atypical, but previously described, features in one eye which showed non-granulomatous chronic lymphocytic infiltration of the choroid and loss of choriocapillaris architecture. ${ }^{16-19}$

Enucleation of the exciting eye was not related to better visual outcome. In addition, the contributing ophthalmologists did not attribute any resolution of disease activity to enucleation, with no significant difference in the maintenance CST dose ( $\mathrm{mg} / \mathrm{kg} /$ day) required to control disease activity between patients who had an enucleation $(0.10(0.04))$ and those who did not have enucleation of their injured eye $(0.16(0.12))$

\section{Discussion}

This prospective population based study of sympathetic ophthalmia confirms the very low incidence of SO. Furthermore, current data demonstrate that ocular surgery, particularly retinal surgery, is now a greater risk for SO than accidental trauma. Compared with previous older studies ${ }^{12-14} 19$ where SO was more likely to occur in males, in children and the elderly, this study shows no sex predominance and a lower risk for children, probably because of the reduced role of accidental trauma and 
reduced incidence of eye injuries in children. ${ }^{20}$ The increased SO risk in the elderly in this current study is likely to be because of the increased frequency of ocular surgery, and possibly because of the increased frequency of retinal detachment in the elderly. ${ }^{21}$ Two of the most recent reports, retrospective case series of 32 patients from the National Eye Institute, $\mathrm{USA}^{7}$ and 18 patients from Moorfields Eye Hospital, ${ }^{14}$ showed that ocular surgery was the sole cause of SO in nine $(28 \%)$ and three $(17 \%)$ patients respectively compared with the current figure of $10(56 \%)$ patients. There is increasing evidence that infiltrating lymphocytes from both the eye and peripheral blood are activated and can proliferate to retinal antigens in patients with SO. ${ }^{9}{ }^{10}$ One may speculate that retinal surgery itself, particularly in susceptible individuals, ${ }^{22}$ is enough to sensitise peripheral lymphocytes to putative retinal autoantigens.

Early reports suggested that sympathetic ophthalmia occurred in $80 \%$ patients within 3 months of injury. ${ }^{623}$ Our data show that only $33 \%$ patients currently develop SO within 3 months of initial injury, with less than half our patients developing SO within 1 year of injury. These findings are similar to the Moorfields series, ${ }^{14}$ and suggest that the time interval between initial injury and disease onset may be longer than traditionally thought.

The clinical features of SO at presentation in this study are in broad agreement with previous reports. ${ }^{67121419}$ Most patients (11, $61 \%$ ) presented with a moderate or severe bilateral panuveitis. By study definition, patients with bilateral anterior uveitis alone were excluded owing to possible diagnostic uncertainty, but this has been previously reported as an atypical finding of $\mathrm{SO}$ at presentation. ${ }^{19}$ Posterior or intermediate uveitis alone was seen in only one $(6 \%)$ patient. Dalen-Fuchs nodules, a typical feature but not essential for diagnosis, ${ }^{14}$ were present at presentation in nearly half our patients. Both choroidal neovascularisation and extraocular signs (sensorineural deafness) are rare but well recognised features of SO. ${ }^{5624}$

Perhaps the most important finding of current surveillance of SO is that the visual prognosis is good, and better than older studies. $^{7}{ }^{12-1419}$ Three quarters of patients achieved visual acuity of $6 / 12$ or better at 1 year and the worst visual acuity was $6 / 60$. Our data confirm that good visual prognosis is related to prompt and adequate systemic immunosuppression, as shown in the National Eye Institute series. ${ }^{7}$ It was encouraging to note that $11(61 \%)$ of 18 patients received systemic corticosteroids within 2 weeks of symptom onset at an adequate dose, and subsequently $11(69 \%)$ of 16 patients followed up received additional immunosuppression with cyclosporin A or azathioprine.

Although prospective, this study was confined to surveillance of Britain and Ireland, and recruited too small a number of patients to definitively ascertain the role of enucleation in the management of sympathetic ophthalmia. It appears that enucleation of the exciting eye is less likely to be performed than previous reports, with $10(50 \%)$ enucleations in 20 patients recruited between 1974 and 1985 in one study ${ }^{13}$ and $19(59 \%)$ enucleations in 32 patients recruited between 1982 and 1992, ${ }^{7}$ compared with five $(28 \%)$ enucleations in the current study. Enucleation as a therapeutic intervention to reduce SO activity appears to be less frequent, with only two of five performed for this indication in this study.

Interpretation of the findings of this study, particularly incidence estimation, must also take account of the fact that the average response rate over 12 months was $64 \%$, less than desirable. The level of acceptable response depends largely on how atypical are the non-responders in a questionnaire. ${ }^{25}$ While it seems unlikely that a newly diagnosed case of sympathetic ophthalmia would not be reported, owing to its rarity and possibly devastating visual consequences, case ascertainment relied on voluntary reporting of $\mathrm{SO}$, a dreaded rare complication of ocular surgery which some ophthalmologists may have been reluctant to report. Other reasons for not reporting a new case could include misdiagnosis (present in only one patient in our cohort), forgetfulness, or unwillingness to contribute data. Overdiagnosis is less likely as all patients had been diagnosed by either a uveitis or medical retina subspecialist. Therefore the current sympathetic ophthalmia incidence figure of $0.03 / 100000$ is likely to be a minimum estimate.

Presented in part at the Annual Meeting of the Association for Research and Vision in Ophthalmology, USA, May 1999.

This project was made possible by the data contribution of 20 ophthalmologists throughout the UK and Republic of Ireland which the authors wish to gratefully acknowledge. The authors which the authors wish to gratefully acknowledge. The authors would like to acknowledge the support of the Wellcome Trust, which supports Dara Kilmartin with a Vision Research Training Fellowship, and the Iris Fund for Prevention of Blindness, which supports the British Ophthalmological Surveillance Unit. BOSU and Ms Parul Desai, Institute of Ophthalmology, BOSU and Ms Parul Desai,
London for helpful discussions.

1 Allen JC. Sympathetic ophthalmia: a disappearing disease. FAMA 1969;209:1090.

2 Liddy BSTL, Stuart J. Symathetic ophthalmia in Canada. Can f Ophthalmol 1972;7:157-9.

3 Kraus-Mackiw E, Muller-Ruchholtz W. Sympathetic eye disease: diagnosis and therapy. Klin Monatsbl Augenheilkd 1980;176:131-9

4 Gass JD. Sympathetic ophthalmia following vitrectomy. Am 7 Ophthalmol 1982;93:552-8.

5 Chan C-C, Roberge FG. Sympathetic ophthalmia. In: Pepose JS, Holland GN, Wilhelmus FR, eds. Ocular infection and immunity. St Louis: Mosby Year Book, 1996; Chapter 57:724.

6 Nussenblatt RB, Whitcup SM, Palestine AG, eds. Sympathetic ophthalmia. In: Uveitis: fundamentals and clinical practice. 2nd ed. St Louis: Mosby, 1996:306-7.

7 Chan C-C, Roberge FG, Whitcup SM, et al. Thirty-two cases of sympathetic ophthalmia: a retrospective study at the National Eye Institute, USA from 1982-1992. Arch Ophthalmol 1995;113:597-600.

8 Forrester JV. New concepts on the role of autoimmunity in Forrester JV. New concepts on the role of autoin
the pathogenesis of uveitis. Eye 1992;6:433-46.

9 Marak GE Jr, Font RL, Johnson MC, et al. Lymphocytestimulating activity of ocular tissues in sympathetic ophthalmia. Invest Ophthalmol Vis Sci 1971;10:770-4.

10 Liversidge J, Dick A, Cheng Y-F, et al. Retinal antigen specific lymphocytes, TCR-gamma delta T cells and CD5+ B cells cultured from the vitreous in acute sympathetic ophthalmitis. Autoimmunity 1993;15:257-66.

11 Chan C-C, BenEzra D, Rodrigues MM, et al. Immunohistochemistry and electron microscopy of choroidal infiltrates and Dalen-Fuchs nodules in sympathetic ophthalmia. Ophthalmology 1985;92:580-90.

12 Albert DM, Diaz-Rohea R. A historical review of sympathetic ophthalmia and its epidemiology. Surv Ophthalmol 1989;34:1-14.

13 Jennings T, Tessler HH. Twenty cases of sympathetic ophthalmia. Br f Ophthalmol 1989;73:140-5. 
14 Hakin KN, Pearson RV, Lightman SL. Sympathetic ophthalmia: visual results following modern immunosuppressive therapy. Eye 1992;6:453-5.

UK, 1997.

16 Fuchs E. Über sympathisierende Entzundung zuerst Bemerkungeen uber seröse traumatische Iritis. Albrecht von Graefes Arch Ophthalmol 1905;30:280-5.

17 Rao NA, Robin J, Hartman D, et al. The role of the penetrating wound in the development of sympathetic ophthalmia: experimental observations. Arch Ophthalmo 1983;101:102-4.

18 Croxatto JO, Rao NA, McLean IW, et al. Atypical histopathologic features in sympathetic ophthalmia: a study of a hundred cases. Int Ophthalmol 1981;4:129-35.

19 Lubin JR, Albert DM, Weinstein DM. Sixty-five years of sympathetic ophthalmia: a clinicopathologic review of cases (1913-1978). Ophthalmology 1980;87:109-21.
20 MacEwen CJ, Baines PS, Desai P. Eye injuries in children: the current picture. Br F Ophthalmol 1999;83:933-6.

21 Rowe JA, Erie JC, Baratz KH, et al. Retinal detachment in Olmsted County, Minnesota, 1976 through 1995. Ophthalmology 1999;106:154-9.

22 ShindoY, Ohno M, Usui $\mathrm{H}$, et al. Immunogenetic study of sympathetic ophthalmia. Tissue Antigens 1997;49:111-15.

23 Marak GE Jr. Recent advances in sympathetic ophthalmia. Surv Ophthalmol 1979;24:141-56.

24 Kilmartin DJ, Forrester DJ, Dick AD. Cyclosporineinduced resolution of choroidal neovascularization in sympathetic ophthalmia. Arch Ophthalmol 1998;116:24950.

25 Coggon D, Rose G, Barker DJP, eds. Planning and conducting a survey. In: Epidemiology for the uninitiated. 4th ed. London: BMJ Publishing Group, 1997:27-36. 